\title{
Le Premier homme en perspective, textes réunis et présentés par Raymond Gay-Crosier
}

\section{Emanuele Kanceff}

\section{Q OpenEdition \\ 1 Journals}

Edizione digitale

URL: https://journals.openedition.org/studifrancesi/38292

ISSN: 2421-5856

\section{Editore}

Rosenberg \& Sellier

\section{Edizione cartacea}

Data di pubblicazione: 15 décembre 2004

Paginazione: 648-649

ISSN: 0039-2944

\section{Notizia bibliografica digitale}

Emanuele Kanceff, «Le Premier homme en perspective, textes réunis et présentés par Raymond GayCrosier», Studi Francesi [Online], 144 (XLVIII | III) | 2004, online dal 30 novembre 2015, consultato il 08 mai 2021. URL: http://journals.openedition.org/studifrancesi/38292

Questo documento è stato generato automaticamente il 8 mai 2021.

\section{(c) (1)}

Studi Francesi è distribuita con Licenza Creative Commons Attribuzione - Non commerciale - Non opere derivate 4.0 Internazionale. 


\title{
Le Premier homme en perspective, textes réunis et présentés par Raymond Gay-Crosier
}

\author{
Emanuele Kanceff
}

\section{NOTIZIA}

AA. VV., Le Premier homme en perspective, textes réunis et présentés par Raymond GAYCROSIER, «Albert Camus», 20, Paris-Caen, Lettres Modernes Minard, 2004, 206 pp. («La Revue des Lettres Modernes» collection fondée et dirigée par Michel MINARD. Éditeur de la Série ALBERT CAMUS: Raymond Gay-Crosier).

1 Questo numero della rivista, che si mostra particolarmente ricco, é suddiviso in tre parti. Dopo una prefazione di Raymond GAY-CROSIER (Camus urbi et orbi), il volume si dipana su parti monografiche, la prima dedicata a Le Premier homme, la seconda alla ricezione di Camus nei Paesi dell'Est, la terza a studi sul nihilismo e sui rapporti Nietzsche-Camus, nel modo seguente:

2 Rumond GAY-CRosier, Camus urbi et orbi (pp. 3-12); I. Le premier homme: Yosei MATSUMOTO, «Le Premier homme»: le processus d'élaboration (pp. 15-32); Christy LAWRENCE, «Le Premier homme»: l'aube de Némésis (pp. 33-62); Geraldine F. MONTGOMERY, La Mère sacrée dans «Le Premier homme» (pp. 62-86); Yosei матsимото, L'Ombre portée par «Le Premier homme» sur «L'exile et le royaume» (87-100); II. La Réception de Camus dans les pays de l'est. Isabelle CIELENS, La Réception de Camus en Lettonie (pp. 105-132); Andor HORVÁTH, «Restituer Dieu à lui-méme»: lectures hongroises d'Albert Camus (pp. 133-148); Jana ратоскоVA, Camus sur les scenes tchèques dans les années Soixante (pp. 149-172); III. Études. Samanthe Novello, Du nihilisme aux théocraties totalitaires: «Les Sources et le sens du communisme russe» de Berdiaev dans les «Carnets» de Camus (pp. 175-196); Frantz FAVRE, Quand Camus lisait Nietzsche (pp. 197-206). 
Questo frammento di romanzo, che è una velata autobiografia, è anche per lo scrittore l'occasione per raccontare le angosce dell'emigrazione e le migrazioni incessanti di una colonia in crisi. Le proporzioni epiche che ne conseguono divengono l'integrazione di esperienze personali e di procedimenti narrativi esperimentati nei romanzi precedenti.

Questa tendenza di Camus a universalizzare i suoi temi romanzeschi è certamente all'origine di una accoglienza attenta nel mondo intero, come ben mostrano sia le molte traduzioni che di questo romanzo sono state ben presto fatte, sia l'attenzione universale della critica, che bene si esprime nella riflessione internazionale che questo volume rappresenta.

Grazie a numerose annotazioni prese dai Carnets, Yosei Matsumoto ricostruisce il processo d'elaborazione de Le Premier homme. Lo studioso propone l'ipotesi che la concezione concreta del romanzo risalga all'ottobre 1953 e coincida con i quarant'anni di Camus, epoca che Matsumoto considera "une sorte de charnière de son travail et de sa vie". Fra il 1953 e il 1956 si trovano negli scritti di Camus numerose allusioni a questa opera futura. Ma fin dal 1942 alcune note di Camus producono una serie di episodi e di annotazioni delle quali Matsumoto raccoglie e recensisce meticolosamente le varie apparizioni. Inoltre, una terza fonte testuale si trova nel progetto di prefazione a L'Envers et l'endroit del 1949 e più particolarmente in una prima allusione a questo progetto di prefazione che data del maggio 1937 e che deplora le imperfezioni formali dei primi saggi.

6 Christy Lawrence si occupa dell'apparire di Nemesi, partendo dalla iperstruttura che, secondo lo stesso Camus, regge l'organizzazione della sua opera, particolarmente il mito di Nemesi, che dopo quelli di Sisifo e di Prometeo doveva formare il centro del terzo pannello della sua concezione. Nemesi fa la sua prima apparizione già nella tesi di laurea di studi superiori, che data del 1936, ma dominerà soprattutto l'ultimo decennio della vita di Camus, costituendo come una specie di prolungamento della «pensée de Midi». Le Premier homme è una ricerca dell'innocenza e dell'unità che né la ricerca del padre, né il pellegrinaggio alle fonti potranno produrre.

7 Constatando che la bozza dell'ultimo romanzo di Camus «rassemble, en les éclairant d'une lumière nouvelle, la plupart des aspects familiers du maternel camusien», Geraldine Montgomery esamina il ruolo della "Madre sacra" nel romanzo, al fine di fame risaltare «le pouvoir structurant de la relation mère-enfant».

8 I rapporti intertestuali tra le novelle di Camus e il progetto di romanzo costituiscono l'oggetto di un ulteriore studio di Matsumoto, che porta il titolo L'Ombre de "Le Premier homme" sur "L'Exil et le royaume". Lo studioso segue una pista aperta da Roger Quilliot $\mathrm{e}$ dichiara che, se la genesi evidente nei Carnets indica chiaramente che queste due opere sono state concepite da Camus come autonome, tuttavia la loro rassomiglianza tematica, geografica e cronologica è stupefacente. La presenza della raccolta di novelle nel futuro romanzo è anche provata da una risposta che Camus ha dato durante un' intervista, quando ha parlato delle novelle come di opere di transizione destinate, secondo Matsumoto, a sfociare logicamente nel romanzo in preparazione.

9 Nella seconda parte prendono posto gli studi sulla ricezione dell'opera di Camus nei Paesi dell'Est.

10 Isabelle Cielens studia la difficile ricezione delle opere di Camus in Lettonia, sotto un regime politico influenzato dal clima repressivo dell'occupazione russa, a partire dal 1940 e fino al recente ritorno della democrazia. Tale regime ha causato la soppressione 
quasi totale della libera espressione e la mancata fortuna delle opere è continuata in seguito a causa di condizioni finanziarie e culturali poco propizie alla diffusione del libro e alla lettura.

11 In Ungheria, sotto il regime di Kadar all'indomani della protesta degli intellettuali contro l'invasione russa, il pubblico potrà finalmente cominciare a leggere Camus, come Andor Horvath precisa all'inizio della sua analisi della fortuna ungherese delle opere di Camus. Il romanziere francese è lungamente conosciuto come scrittore di fiction piuttosto che come saggista e filosofo: le opere controverse, in particolare L'Homme revolté, erano tuttavia accessibili alla ristretta cerchia di intellettuali francofoni, sicché è possibile parlare di una influenza immediata e intima di Camus nell'evoluzione della letteratura ungherese.

12 Nella terza parte, "Études", si situano ancora due testi. Samanta Novello (Du nihilisme aux théocraties totalitaires) riflette in chiave politica su Carnets II e afferma che bisogna cercare in quest'opera le fonti nascoste delle letture che Camus ha potuto fare di Berdiaev. La studiosa ci fornisce l'inventario completo dei passi nei quali Camus ha scritto letteralmente, parola per parola, frasi di quest'ultimo autore.

Franz Favre, infine, presenta un inventario utilissimo dei libri di Nietzsche e su Nietzsche che si trovavano nella biblioteca personale di Camus; ma il suo lavoro è anche e soprattutto una recensione di citazioni generalmente molto fedeli della Volonté de puissance che Camus riprende ne L'Homme revolté. 